Assurances et gestion des risques

Insurance and Risk Management

\title{
Microassurance santé : un état de l'art. Une revue du livre \\ Financing Micro Health Insurance - Theory, methods and evidence par David M. Dror
}

\section{Michel Vaté}

Volume 86, numéro 1-2, juin 2019

URI : https://id.erudit.org/iderudit/1062467ar

DOI : https://doi.org/10.7202/1062467ar

Aller au sommaire du numéro

Éditeur(s)

Faculté des sciences de l'administration, Université Laval

ISSN

1705-7299 (imprimé)

2371-4913 (numérique)

Découvrir la revue

Citer ce document

Vaté, M. (2019). Microassurance santé : un état de l'art. Une revue du livre Financing Micro Health Insurance - Theory, methods and evidence par David M. Dror. Assurances et gestion des risques / Insurance and Risk Management, 86(1-2), 105-116. https://doi.org/10.7202/1062467ar

Tous droits réservés (C) Faculté des sciences de l'administration, Universite Laval, 2019
Ce document est protégé par la loi sur le droit d'auteur. L'utilisation des services d'Érudit (y compris la reproduction) est assujettie à sa politique d'utilisation que vous pouvez consulter en ligne. 


\section{MICROASSURANCE SANTÉ : UN ÉTAT DE L'ART UNE REVUE DU LIVRE FINANCING MICRO HEALTH INSURANCE - THEORY, METHODS AND EVIDENCE PAR DAVID M. DROR}

Michel VATÉ1

"La santé pour tous à des prix abordables", la formule claque un peu comme un slogan. Elle aurait pu être le sous-titre du plus récent ouvrage de David M. Dror qui est intitulé: Financing Micro Health Insurance - Theory, Methods and Evidence ${ }^{2}$. Elle donne un cap.

\section{POURQUOI CE LIVRE? POURQUOI MAINTENANT?}

Laissons l'auteur nous dire lui-même quelle est sa réponse à la première de nos deux questions: "Ce livre aborde trois questions. La première est comment catalyser la demande pour l'assurance maladie et développer l'éducation à l'assurance au sein de la population cible qui, en grande partie ne sait ni écrire ni compter, en utilisant des programmes de formation pour construire un consensus qui permette localement aux gens de lancer et administrer eux-mêmes de tels systèmes.

La seconde consiste à élaborer des méthodes simplifiées d'évaluation des risques, ce qui est indispensable pour souscrire des risques, établir le prix de la microassurance santé, et assurer la mise en ouvre correcte.

Le troisième sujet est de formuler une analyse de rentabilisation convaincante qui rendrait cette assurance abordable, financièrement viable, et de plus grande envergure sur le plan opérationnel». Un tel 
exercice n'était pas concevable sans le background de vingt années d'immersion dans l'univers empirique et académique de la microassurance santé (MHI).

En effet, ces deux questions n'en font qu'une en réalité : pourquoi ce livre? parce que le moment était venu de dresser un état de l'art. C'était nécessaire et c'était possible. L'auteur répond à cette question double en cinq cents pages, très sérieuses et parfois ardues, mais toujours stimulantes pour quiconque s'intéresse à ce "jeune» domaine de recherche qu'est la MHI.

Oui, la microassurance a vingt ans. Les experts, les chercheurs et les professionnels du développement et de l'assurance qui se sont intéressés très tôt à cet objet qui était encore mal identifié se souviennent des réserves et des doutes qui ont salué son apparition. Était-il vraiment utile d'inventer la MHI? Cela ne faisait-il pas double emploi avec d'autres instruments? Était-ce applicable auprès de populations pauvres? Les deux premières questions, utilité et spécificité de la MHI, ont été tranchées très tôt. Après vingt ans d'études, d'enquêtes, d'expériences et de conférences - dont, par exemple, les 14 éditions de la Microinsurance Conférence (Munich Re Foundation) sont une sorte de conservatoire - ce livre arrive a point pour dire où l'on en est sur la troisième question, primordiale, de la viabilité de la MHI dans des contextes qui sont a priori défavorables. Comme souvent, la réponse tient en trois mots : rigueur, résilience et chance. Par hypothèse, le dernier facteur est hors de contrôle - à l'exception des actions de prévention qui affectent les conditions de fonctionnement du mécanisme assurantiel, mais qui lui sont extérieures - et l'avenir dépend donc de la qualité des efforts que l'on veut bien concentrer sur les deux autres.

Vingt ans, c'est trop long pour demeurer dans une attitude d'étonnement - enthousiaste ou sceptique, c'est selon - face à la nouveauté. Vingt ans, c'est sans doute trop court pour aboutir à la connaissance théorique et méthodologique complète d'un nouvel objet scientifique que la pratique n'a pas encore pleinement assimilé ni, a fortiori, validé. Mais ce livre apporte la preuve que vingt ans, c'est suffisant pour engager solidement la construction d'une telle connaissance dans une voie qui soit socialement utile et scientifiquement rigoureuse. Dans le monde économique, tout nouveau système est potentiellement viable dès lors qu'il possède trois propriétés au moins: logique interne, compatibilité externe avec le système environnant, utilité commune. Il reste alors, et c'est beaucoup, à affronter l'épreuve de l'expérience. Parce que la MHI est à base communautaire et parce que "comme les 
assureurs commerciaux, les MIU s'efforcent également de payer toutes les demandes d'indemnisation souscrites en tout temps" (p. 225), personne n'a intérêt à pérenniser une expérience de MHI qui ne serait pas satisfaisante, ni pour le bien-être des personnes engagées, ni au regard de l'exigence minimum de viabilité financière.

\section{REVENONS VINGT ANS EN ARRIÈRE}

À la fin des années 1990, le microcrédit est déjà bien installé dans le paysage financier des pays à bas revenu, et il occupe une large place jusque dans les médias destinés au grand public. On commence à oublier le scepticisme, les critiques ou même l'hostilité qui, avant d'en arriver là, avaient dû être surmontés par ses inventeurs, Muhammad Yunus au premier rang, puis tant d'autres qui l'ont suivi. Bientôt, on pourra parler de finance inclusive sans que cela paraisse utopique: portées par le succès du microcrédit, et souvent en liaison avec celui-ci, d'autres branches de la microfinance (bancarisation, épargne) ont pu être développées car le plus dur était fait. Restait le cas de l'assurance, ô combien plus difficile. À cette époque, on ne parle pas encore de microassurance. Quand on parle d'assurance, c'est pour voir en elle un produit de luxe dont la diffusion viendra avec le développement: c'est la fameuse courbe en $\mathrm{S}$ de la pénétration de l'assurance (primes en \% du PIB) par rapport au PIB par habitant. Quant à la couverture du risque santé, elle n'est pas imaginée autrement que sous la forme de systèmes publics de protection sociale dont la mise en ouvre se heurte, sur le terrain, à des obstacles techniques et pratiques, plus encore que financiers. Et c'est justement dans le domaine de l'assurance santé que le concept de microassurance va faire son apparition, en dehors du monde du microcrédit.

En effet, David Dror et Christian Jacquier publient en 1999 un article 3 qui propose "a concept for group-based health insurance, or microinsurance». L'objectif est clair: "Étendre l'assurance maladie aux exclus ", et les contours du projet sont déjà précis : "la microassurance est conçue comme une entreprise autonome, indépendante d'opérateurs externes ou de soutiens financiers permanents" et "la microassurance est l'entreprise de la communauté». Une histoire commence. Très vite, des expériences pilotes se multiplient (Ouganda, Philippines...), la littérature explose (articles théoriques, retours d'expériences...) et les conférences régionales ou internationales se multiplient. 
À mi-parcours, en 2009, soit deux ans après avoir créé la Micro Insurance Academy à New Dehli, David Dror est nommé "personnalité de l'année" par l'Asia Insurance Industry. Le livre Financing Micro Health Insurance qu'il publie maintenant est donc l'œuvre d'un pionnier qui n'a pas quitté la ligne de front depuis vingt ans, et cela fait de cet ouvrage une major Milestone dans la (jeune) histoire de la microassurance.

\section{FINANCER QUOI? ET COMMENT?}

Financing, insurance, theory... Au premier abord, le titre du livre semble annoncer un sujet extrêmement technique, peut-être austère. Et cette impression pourrait être renforcée par la rigueur méthodologique et l'usage intensif des outils économétriques. Mais, chapitre après chapitre, des questions essentielles se bousculent et plongent le lecteur dans la vraie vie: financing? oui, mais pourquoi? pour qui? quoi? comment? Et là on croise des hommes et des femmes, des communautés, des conditions d'existence, des calculs, des hésitations et des angoisses, mais aussi des projets portés par le refus des fatalités. Sous nos yeux, c'est tout un pan de l'Économie du développement qui est en train de s'écrire, et cela concerne de vastes "zones blanches" (uncovered areas) de la macroéconomie du développement, comme de la microéconomie de l'assurance.

Était-il bien nécessaire "d'inventer" la microassurance santé? demandions-nous plus haut. Le sujet n'est pas mineur. Voilà plus de 70 ans que la Déclaration Universelle des Droits de l'Homme proclame un droit à la santé et à la protection sociale (art. 25). S'agissant précisément de la couverture du risque santé, trois schémas sont envisageables et ont été expérimentés à travers le monde: groupements locaux de solidarité vernaculaire, sur une base familiale, villageoise ou professionnelle; entreprises d'assurances; système public de sécurité sociale. En de très nombreux endroits du monde, on a le choix entre les trois méthodes et l'on peut même imaginer de les combiner, parce que les conditions de viabilité qui sont requises par chacune d'elles sont (à peu près) convenablement remplies. Ailleurs, hélas!, les revenus sont trop faibles, l'éducation trop incomplète, les outils de la finance inclusive trop éloignés, et les rouages de l'action publique trop imparfaits: il faut imaginer autre chose qu'un upsizing des groupements locaux (pouvoir bénéficier de la loi des grands nombres est chèrement payé par les coûts administratifs et la perte du contrôle social), ou autre chose qu'un downsizing des assurances commerciales (la diminution 
des primes ne compense pas le prix du capital de sécurité); quant aux systèmes publics, ils se heurtent à des difficultés insurmontables dans la mise en œuvre concrète de leur caractère obligatoire et universel.

Si l'on dressait une carte de l'application de l'art. 25 dans le monde, on verrait immédiatement que celle-ci laisse encore apparaître de nombreux trous. La démonstration de $\mathrm{D}$. Dror est que ces trous peuvent être réduits en partant du modèle de la community-based health insurance, mais à condition de ne pas faire n'importe quoi: la CBHI est d'abord une assurance. Par conséquent, elle doit respecter les règles de l'assurabilité, elle doit caler sa tarification sur le consentement à payer, elle doit accepter la "tyrannie» du coefficient de sécurité... Défini comme le taux de couverture des pertes probables par le montant des fonds propres, le coefficient de sécurité fait irruption dans la gestion des risques dès que l'on quitte le cadre (forcément réduit) d'un accord équitable de réciprocité dans le partage du risque, pour entrer dans le cadre, plus anonyme, du transfert de risque. Ceci n'est pas une exigence des actionnaires (il n'y en a pas!); c'est l'intérêt bien compris des personnes affiliées. C'est aussi une exigence d'intérêt général : dans la mesure où le fait d'être assuré sur la santé a un impact sur les comportements économiques des affiliés qui tablent sur une sûreté accrue (chapitres 20 et 21), il serait catastrophique que leurs plans soient ruinés par la défaillance financière de l'unité d'assurance. On pense en particulier à la réduction de l'épargne de précaution, au profit de choix de production plus efficients et, pourquoi pas?, de la souscription d'une microassurance sur les récoltes agricoles! David Dror nous engage à garder les pieds sur terre: dans les lieux où les conditions ne sont pas réunies pour que l'extension de la couverture sanitaire puisse procéder de l'action gouvernementale, ne rêvons pas, la MHI ne peut être une solution soutenable que si, et seulement si, elle respecte une discipline stricte.

\section{UN UNIVERS PLUS STATISTIQUE QUE MACROÉCONOMIQUE}

De la première à la dernière page, le texte se déploie dans une "ambiance" résolument statistique, que l'on pourrait situer quelque part entre macro- et microéconomie. Les agrégats macroéconomiques sont peu présents, et ils apparaissent bien dans leur rôle habituel de références qui permettent d'étalonner les phénomènes étudiés. Quant à la théorie microéconomique de la demande, une large place lui est faite dans la première partie, mais c'est essentiellement pour constituer un support rigoureux aux analyses statistiques qui viendront ensuite. 
Le caractère "statistique" de la démarche est particulièrement illustré par le chapitre 8 et le modèle qu'il propose: "La connaissance de la distribution des coûts de santé est essentielle afin d'ajuster les coûts au consentement à payer pour l'assurance santé, et dans le but de prévoir (par simulation) la performance attendue de la microassurance santé", écrit l'auteur. No less. De ce modèle, on peut tirer deux enseignements très intéressants.

En premier lieu, l'étude montre un très bon ajustement des données avec le modèle log-normal. En théorie, cela signifie que les facteurs déterminants sont nombreux et de faible intensité individuelle, qu'ils sont indépendants entre eux, et que leurs effets sont multiplicatifs (et non pas additifs, comme ce serait le cas pour une distribution normale). Ces quatre caractères sont importants, mais le plus important est sans doute le dernier, qui met en évidence ce que les statisticiens appellent une loi de l'effet proportionnel: on en comprend immédiatement l'importance pour le sujet qui nous intéresse ici, aussi bien pour la compréhension du phénomène étudié (répartition des coûts de santé) que pour les usages prédictifs du modèle (calibrage de l'équilibre financier).

La seconde leçon est tirée de la comparaison d'une dizaine de distributions locales: dans l'ensemble, elles sont très similaires les unes aux autres, sauf à la queue gauche, c'est-à-dire pour les petites valeurs. Rappelons d'abord que l'histoire moderne de la théorie du risque nous enseigne que ce qui se produit aux extrémités d'une distribution est souvent plus instructif que le cour de celle-ci. Mais il y a plus. On devrait toujours s'intéresser aux "désordres" qui affectent la left tail d'une distribution (ou la right tail selon les cas), même lorsque celle-ci est très bien ajustée par ailleurs, car cela peut avoir un sens, et même un sens profond en rapport avec les phénomènes sous-jacents. De telles anomalies sont rares dans les applications naturelles ou techniques du modèle log normal (granulométrie, hydrologie, fiabilité), là où prévalent les lois de la physique. Elles sont plus fréquentes dans les données relatives aux systèmes économiques et sociaux, telles que les revenus, la taille des unités de production, la dimension des villes, le cours de titres financiers, etc.: elles signifient que les écarts d'ajustement ne sont pas répartis de manière aléatoire sur l'étendue totale de l'intervalle de variation. Alors, si l'explication ne se trouve pas dans le hasard pur, il faut aller chercher celle-ci du côté des altérations de comportement ou de la violation de la condition d'indépendance, et cela est fondamental pour l'analyse et pour l'action. 


\section{UN OBJET EXTRÊMEMENT COMPLEXE}

Micro + assurance + santé, en combinant ces trois mots on crée un objet qui est d'une rare complexité.

Micro - qui est entendu ici dans le sens de community-based et non d'atomistique - désigne le niveau d'analyse qui, en économie, est toujours le plus complexe, celui des échelles intermédiaires entre l'individuel et le global, entre un (le producteur) et tous (la production nationale): c'est le domaine des coalitions, de la coopération, des mesures partiellement agrégées, de la méso-analyse, de l'articulation entre grandeurs statistiquement distribuées, etc. Consacré au thème de l'impact, le chapitre 20 illustre ce point en montrant comment le comportement global dépend de la taille (inégale) des groupes intermédiaires, de leur capacité financière (inégale), de leur degré de cohésion interne, ou encore de leur stabilité.

Assurance désigne peut-être l'opération la plus complexe parmi toutes les opérations économiques. Elle est, tout à la fois, un outil de gestion des risques, un produit financier, impensable sans le grand nombre, un arbitrage intertemporel, l'achat d'un service, un espace de solidarité, etc. Un crédit peut être complètement défini entre deux personnes seulement, le prêteur et l'emprunteur, sur le modèle de la reconnaissance de dette; une personne peut être solidaire d'une autre personne mais elle ne peut pas, à elle seule, en être l'assureur. Partage et transfert de risque sont inhérents à l'assurance.

L'industrie moderne de l'assurance repose aujourd'hui sur un corpus théorique et méthodologique considérable; elle bénéficie des leçons d'un apprentissage qui s'étire sur plusieurs siècles, qui fut aidé par l'innovation financière et qui est enrichi aujourd'hui par l'irruption du big data. Il serait temps que tout cela profite aussi aux populations les moins favorisées dans les pays les plus pauvres.

Santé, enfin, désigne le bien le plus difficilement classable dans les nomenclatures économiques usuelles, car c'est un bien sans demande, sans offre, sans marché et donc sans prix, mais c'est quand même un "bien" qui a une valeur - pour l'individu et pour la collectivité, et ce n'est pas la même dans les deux cas - et qui coûte, parfois très cher. Certes, on peut assez facilement faire entrer les soins et les actions de prévention dans le cadre des analyses de type micro-économique. Mais l'exercice est déjà plus malaisé pour les externalités, ce qui rend particulièrement ardue la couverture assurantielle du risque épidémique. Et il s'avère quasi-impossible pour la santé quand elle est regardée 
comme une composante du capital humain, comme un pilier - avec l'instruction - de ce que Amartya Sen appelle la "capabilité", ou comme un indicateur entrant dans la mesure du développement humain d'une collectivité.

On croise cette multitude de sources de complexité presqu'à chaque page du livre de David Dror: placé en face d'elle, l'auteur ne choisit ni la fuite, ni le renoncement, mais l'affrontement. Le résultat est que cela demande aussi un petit effort... au lecteur! En prime, comme si le sujet n'était pas déjà assez complexe, on ne peut pas bien comprendre ce qu'est vraiment la richesse du concept de MHI si on voit en elle seulement une assurance-maladie unidimensionnelle. Elle est aussi une assurance-emprunteur, une assurance-producteur, une assurancechef de famille, etc., parce que le souscripteur est tout cela à la fois et qu'aucune de ces dimensions ne peut être traitée indépendamment des autres. Dans la mesure où elle prend en charge la vulnérabilité aux risques primaires contre laquelle les producteurs pauvres sont démunis, la MHI combat le principal handicap qui fait obstacle à la sortie de la pauvreté. Mieux, en impliquant les acteurs dès le début du processus, la stratégie de protection débouche tout naturellement sur une stratégie d'empowerment.

\section{UN SUJET TRANSVERSAL}

Le pire n'est jamais sûr, mais le risque zéro n'existe pas non plus. Et on ne peut pas attendre que les régions pauvres soient devenues riches pour y étendre les solutions qui marchent partout ailleurs. On peut imaginer que le meilleur système de couverture des risques est celui qui sait estimer avec la plus grande précision la distribution du nombre probable des sinistres à couvrir, ainsi que celle du coût probable de chacun de ces sinistres. C'est indispensable, mais pas suffisant. Le système doit anticiper ce que sera sa réponse si la réalité se traduit par une sinistralité extrême. Le défi à relever a deux dimensions, de cohérence interne et de cohérence externe.

La première dimension, interne, est celle de la capacité de supporter les pertes extrêmes, grâce aux fonds propres ou par recours à la réassurance. Le propre de la réassurance, et le problème est bien posé au chapitre 9, est précisément d'endogénéiser la réponse ultime aux situations extrêmes, qui peuvent être des événements extrêmes aussi bien que des cumuls exceptionnels d'évènements banals. En intégrant la réassurance dès le départ, on modifie les paramètres de l'assurance 
primaire avec, à la clé, une meilleure abordabilité du contrat. Cela rejoint la problématique du souscripteur qui redoute d'être confronté, un jour, à une dépense aléatoire qui - pour lui, maintenant, là où il vit et à son niveau de revenu - est perçue comme catastrophique (cf. chapitres 6 et 10).

En supposant que la cohérence interne est satisfaite, la deuxième dimension, externe, est celle de l'intégration micro/macro. Pour que l'extension de l'assurance soit constructive (en termes de contribution au développement macroéconomique), il faut que la souscription corresponde à un comportement rationnel de l'assuré (au niveau microéconomique). Ensuite, l'impact macroéconomique qui est attendu de la MHI (chapitre 1, Box 8 et Part 3) implique que la solvabilité du système soit, dès le départ, intégrée à la conception du dispositif de couverture, et non pas qu'elle reste tributaire, le cas échéant, d'un apport exogène (subvention). Enfin, seule la combinaison de ces deux conditions peut engendrer des externalités systémiques positives: or on sait combien celles-ci sont précieuses pour l'empowerment des agents économiques, comme pour la soutenabilité du processus de développement. Vues sous cet angle, les problématiques respectives du micro-crédit et de la micro-assurance se différencient nettement, mais se complètent. Ainsi commence-t-on à regarder la MHI comme un facteur de soutenabilité de la microfinance en général.

\section{UN TRAITÉ D'ÉCONOMIE APPLIQUÉE}

Ce livre n'est pas - et il ne prétend pas être - un guide pratique, ni un essai théorique. Il est un authentique traité d'économie appliquée, au sens plein et précis du terme. Les avancées théoriques ou méthodologiques n'y sont pas une pure spéculation, mais des réponses rationnelles qui sont apportées à des problématiques clairement identifiées ; les recommandations pratiques n'y sont pas des recettes qui seraient dictées par l'urgence de l'action ou par la facilité, mais bien la projection des outils théoriques sur une réalité convenablement modélisée. Faire une théorie de la MHI et de son financement, cela ne peut pas se limiter à fournir un "mode d'emploi » pour une technique particulière appliquée à un objet particulier.

Une caractéristique remarquable de ce livre est la très abondante bibliographie et l'importance des revues de la littérature (chapitres 5 et 11): elles constituent une aide précieuse pour le chercheur ou l'expert qui est intéressé par ces sujets; elles montrent aussi, au passage, 
que ces sujets intéressent aujourd'hui beaucoup de monde. À chaque endroit, on a sous les yeux les éléments techniques et numériques, ainsi que les définitions, dont on a besoin pour la compréhension du texte; en complément, on sait où trouver une base documentaire plus étendue, ou ce qui serait nécessaire pour un approfondissement du cas étudié. En fonction des besoins propres à chaque sujet étudié, l'auteur propose des ouvertures méthodologiques, par exemple vers l'économie expérimentale (CHAT, real-life experiences...) ou l'économie statistique (travail sur les effets de dispersion ou des indices de concentration, relations entre quantités distribuées à la place d'agrégats...).

\section{MiCROASSURANCE SANTÉ: UN NOUVEL OUTIL POUR LE DÉVELOPPEMENT COHÉSIF}

Tout au long du livre, on remarque l'insistance que David Dror met à expliquer deux choses complémentaires : $1^{\circ}$ pourquoi la confiance, la compréhension, et donc l'éducation, sont si importantes pour hausser la willingness-to-pay pour la MHI malgré la faiblesse des revenus, en tenant compte du fait, essentiel, que le montant de la prime à payer ne doit pas être comparé à zéro, mais au coût des soins parfois élevé que le souscripteur (non assuré) paye déjà sur son faible revenu (cf. chapitre 5); et $2^{\circ}$ pourquoi, en face, la responsabilité des unités d'assurance est d'assumer trois impératifs, à savoir: la simplification et la transparence des procédures, l'évaluation rigoureuse des risques et des gains de bien-être, et le contrôle attentif de l'équilibre entre les coûts (health care costs) et la collecte des primes.

Pour exprimer cela, l'auteur fait abondamment usage d'un vocabulaire qui est celui de l'adhésion: volonté de payer, adhésion volontaire, base communautaire, consensus, gains de bien-être, etc. Tout cela crée chez le lecteur le sentiment que l'adjectif inclusif - aujourd'hui très à la mode - n'est pas suffisant. Certes, les mots inclusion, inclusif, inclusivité, traduisent bien une qualité dont on s'accorde aisément à considérer que, idéalement, elle doit être possédée par tous les outils ou méthodes qui sont appliqués à la résolution des grands problèmes collectifs tels que la lutte contre la pauvreté, la sûreté alimentaire ou le développement humain. Mais, en raison de leur caractère binaire (exclu/inclus), ces mêmes mots s'avèrent insuffisants pour qualifier le fonctionnement d'une collectivité et, a fortiori, celui d'un système humain envisagé dans sa globalité. À sa manière, l'ordre féodal était 
inclusif, tout comme le fut celui des régimes communistes. Les stratégies de développement s'adressent à des individus qui sont vus - ou qui devraient être vus - "comme des acteurs du changement et non comme les destinataires passifs d'avantages octroyés par telle ou telle structure $^{4}$ "(A. Sen). Aussi les institutions qui conduisent ces stratégies ont-elles besoin d'une mesure graduelle pour orienter leurs choix et évaluer les résultats de leurs actions. La propriété d'inclusion ne renseigne pas sur la qualité, l'intensité et le degré d'acceptation de la relation qui unit chaque individu à l'ensemble inclusif. La réponse est dans la cohésion qui tient en une simple équation: Cohésion = Inclusion + Adhésion.

\section{CONCLUSION - ET MAINTENANT?}

En économie appliquée, la recherche et l'expérience s'enrichissent mutuellement de leurs progrès respectifs, et s'interrogent mutuellement sur de nouvelles problématiques. La MHI au service du développement humain n'est pas un champ clos qui serait délimité par une frontière précise. L'état de l'art proposé ici par David Dror constitue un socle solide sur la base duquel quelques questions nouvelles traversent l'esprit du lecteur au moment où il referme ce livre, en même temps qu'il est interpellé par l'actualité scientifique et financière. C'est, par exemple, la question de la qualité et de la précision des données dont on a vu le caractère primordial au fil des chapitres: quel impact pour le big data et le machine learning? les organisations internationales (ILO, FAO, WHO...) n'auraient-elles pas à jouer ici un rôle d'assistance technique d'un nouveau type? C'est aussi la question de la transposabilité des expériences dans l'espace: comment caractériser les populations et les territoires par des indices de spécificité afin d'être guidé vers le choix des modèles les plus appropriés? C'est encore la question de la complémentarité des divers modes d'action, afin qu'ils se complètent en évitant les redondances: comment articuler le rapide déploiement des systèmes locaux de MHI avec la multiplication des fonds d'assurance titrisée ${ }^{5}$ (thématiques ou régionaux) qui sont dédiés aux évènements extrêmes?

Ce n'est pas le moindre mérite du livre de David Dror que de donner à des chercheurs - à commencer par les plus jeunes d'entre eux! l'envie de s'emparer de ces questions. 


\section{NOTES}

1 Professeur émérite à l'Université de Lyon, Ancien Doyen de la Faculté de Sciences économiques de Lyon, Chercheur associé à l'Institut Thomas More (Bruxelles-Paris)

2 Dror, David, M. Financing micro health insurance: Theory, methods and evidence. Hackensack, NJ, World Scientific, 2018. 552 pp. 978-9-813-23849-7.

3 Dror, D.M. and Jacquier, C. (1999). Micro-insurance: Extending health insurance to the excluded, International Social Security Review 52(1): 71-97.

4 Sen, A. (1999). Development as Freedom, Oxford: Oxford University Press, Preface. Traduction française (2000), Un nouveau modèle économique, Paris: Odile Jacob.

5 Clin d'œil de l'histoire, au même moment (printemps 1999) «naissait» la microassurance santé, et était créé aux Bermudes un website, Artemis, avec le but de « fournir un site en ligne pour le secteur émergent des cat bonds (obligations catastrophe) et des ILS (insurance-linked securities), et encourager le développement de la participation des marchés financiers dans la réassurance». Deux mondes qui ont vocation à se croiser dans les nouvelles formes de réassurance des risques extrêmes dont souffrent les pays pauvres. 